\title{
Characteristics and clinical significance of spondylolisthetic and spino-pelvic sagittal parameters in kyphotic low-grade degenerative lumbar spondylolisthesis
}

Chongqing Xu ( $\nabla 09663990 @ q q . c o m$ )

Long Hua Hospital https://orcid.org/0000-0001-9654-793X

Mengchen Yin

Long Hua Hospital

Wen Mo

Long Hua Hospital

Research article

Keywords: degenerative lumbar spondylolisthesis, kyphosis, sagittal parameters, quality of life

Posted Date: November 14th, 2020

DOl: https://doi.org/10.21203/rs.3.rs-107452/v1

License: (a) (1) This work is licensed under a Creative Commons Attribution 4.0 International License.

Read Full License 


\section{Abstract}

Background: Degenerative lumbar spondylolisthesis (DLS) has been a common disease that make increasing patients suffer from different degrees of low back pain and radicular symptoms. At present, the incidence of kyphosis in DLS and the differences in spinal sagittal morphology between kyphotic and non-kyphotic DLS still remain unclear. Our study aimed to determine the incidence of kyphosis in DLS, explore the characteristics of spondylolisthetic and spino-pelvic sagittal parameters, and evaluate the impact of kyphosis on the life quality of patients with DLS.

Methods/Design: All 108 patients with L4/5 low-grade DLS were enrolled and divided into kyphotic and non-kyphotic group. 2 spine surgeons who have certain clinical experience were selected to measure the spondylolisthetic and spino-pelvic parameters, as well as visual analogue scale (VAS) and Oswestry disability index (ODI). Differences between the 2 groups and characteristics of measurements were analyzed.

Results: The inter-observer agreement of all parameters between the 2 spine surgeons were excellent with each ICC > 0.75. Kyphotic group had higher SA, SD, SDM, SAM, PDH, PT and SVA, and lower ADH, SS and $\mathrm{LL}$ than those in non-kyphotic group $(p<0.01)$. There was no significant difference in $\mathrm{PI}$ and TK between the two groups $(p>0.05)$.

Conclusion: The incidence of kyphotic spondylolisthesis in patients with L4/5 low-grade DLS is $16.7 \%$. Compared with those in non-kyphotic group, patients with kyphosis show inferior results of spondylolisthetic parameters, more severe loss of $L L$, more obvious pelvic retroversion and trunk anteversion, which indicates worse spino-pelvic sagittal balance. In addition, their quality of life was significantly lower than that of patients in non-kyphotic group.

\section{Background}

Degenerative lumbar spondylolisthesis (DLS) refers to the lumbar degenerative changes that cause the adjacent vertebral body to slide, while the posterior structure of the vertebral body remains intact, which often occurs in L4/5 segment and make increasing people suffer from different degrees of low back pain and radicular symptoms.[1, 2] The normal L4/5 intervertebral space presents physiological lordosis in sagittal alignment, and the lordotic angle usually ranges from $8^{\circ}$ to $17^{\circ}$.[3] The lordosis of intervertebral space plays an important role in maintaining the whole sagittal balance, especially the lumbar lordosis. The imaging findings of spondylolisthetic segment in DLS include anterior displacement of the spondylolisthesis vertebrae and loss of intervertebral space height, often accompanied by reduced local lordosis or even kyphosis. [4, 5] The typical characteristics of kyphotic DLS are mainly manifested as the anterior collapse of the intervertebral space and the backward opening slip angle.[6, 7]

Kepler et al.[8] proposed the Clinical and Radiographic Degenerative Spondylolisthesis (CARDS) classification based on disc space height, sagittal vertebral translation, kyphotic alignment, and lower extremity pain, of which Type D refers to the kyphosis of the spondylolisthetic segment. The measuring 
of the kyphosis actually reflects the change of the anterior and posterior height of intervertebral disc, however, the spino-pelvis sagittal parameters were not included in the research. Studies have found that the kyphosis caused by the collapse of intervertebral space in patients with DLS is closely related to their quality of life, and indicated that kyphosis is one of the important factors of low back pain. $[9,10]$ With the further study of spinal biomechanics and sagittal balance, the understanding of DLS pathogenic factors and natural history have been increasingly comprehensive.[11]

In recent decades, correlation between spino-pelvic parameters and morbidity of DLS, as well as between spinal sagittal balance and progression of DLS are the hot pots and have been gradually revealed.[12, 13] Nevertheless, at present, the incidence of kyphosis in DLS and the differences in spinal sagittal morphology between kyphotic and non-kyphotic DLS still remain unclear. Thus we retrospectively analyzed the imaging and clinical data of DLS patients with Meyerding classification of grade I-II,[14] and aimed to: 1) determine the incidence of kyphosis in DLS; 2) explore the characteristics of spondylolisthetic and spino-pelvic sagittal parameters in kyphotic DLS; and 3) evaluate the impact of kyphosis on the life quality of patients with DLS.

\section{Materials And Methods}

\subsection{Patient Case Selection}

To perform this study, we obtained institutional review board approval from our ethics committee and informed signed consents were provided by all participating subjects. Database records of patients with DLS treated in our hospital were retrospectively reviewed between January 1, 2017, to June 30, 2020 (as our hospital had updated and used a new medical record system since January 1, 2017). Patients included in the study should be defined as L4/5 single-segment DLS with Meyerding classification of grade I-II, and were required to have complete imaging studies (including full-length spine radiographs, flexion and extension lumbar radiographs, lumbar computed tomography (CT) and three-dimensional reconstruction, and lumbar magnetic resonance (MR) images) and available clinical data (including demographic characteristics, chief complaint, neurological function, medical history, visual analogue scale (VAS) and Oswestry disability index (ODI)). Exclusion criteria were patients with multi-segment spondylolisthesis, previous surgery or trauma history, and combined with other spinal diseases including tumor, tuberculosis, infection, deformity, metabolic bone disease, etc. Finally, a total of 108 consecutive cases in accordance with the criteria were involved in the current study. According to the morphology of the intervertebral space on the standing radiographs, patients were divided into 1) kyphotic group, with larger anterior disc height; and 2) non-kyphotic group, with lower anterior disc height (Fig. 1).[6, 7]

\subsection{Imaging Measurement And Assessment}

A resident of our department who did not participate in the later statistics and analysis collected these cases from the database. 2 independent expert spine surgeons were selected to assess imaging studies and measure parameters separately as they were unaware of the identity of the patients and the 
treatment they received. IDC Cygnus Version 1.2 (DICOM image viewing software) was used to measure sagittal parameters as follows:

Spondylolisthetic parameters: 1) slip degree (SD), measured as the slipped distance of L4 divided by the length of $L 5$ upper endplate; 2 ) anterior disc height (ADH), measured as the distance from $L 5$ anterior upper corner to L4 lower endplate; 3 ) posterior disc height (PDH), measured as the distance from L4 posterior lower corner to L5 upper endplate; 4) slip angle (SA), defined as the angle between L4 lower endplate and L5 upper endplate; 5) slip angle motion (SAM), defined as the absolute value of subtraction between slip angles in radiograph (standing) and T2-weighted MR image (supine); and 6) slip degree motion (SDM), defined as the absolute value of subtraction between slip degrees in radiograph and T2weighted MR image.[15, 16]

Spino-pelvic parameters: 1) lumbar lordosis (LL), measured as the angle subtended between tangents of T12 lower endplate and S1 sacral endplate; 2) sacral slope (SS), measured as the angle subtended between tangent of S1 endplate and horizontal line; 3) pelvic tilt (PT), defined as the angle between the vertical and a line from the center of the femoral heads to the midpoint of the sacral endplate; 4) pelvic incidence (PI), defined as the angle subtended between a line perpendicular to the sacral plate at its midpoint and a line connecting this point to the axis of the femoral heads; 5) thoracic kyphosis (TK), defined as the angle between the perpendicular of T5 upper endplate and the perpendicular of T12 lower endplate; and 6) sagittal vertical axis (SVA), defined as the distance from C7 plumb line to superior corner of the sacral endplate (Fig. 2).[17, 18]

\subsection{Quality Of Life Assessment}

Quality of life assessment was performed based on the statistics and analysis of VAS and ODI scale. Patients were required to fill in the questionnaires at the time of consultation. Another spine surgeon was select to conduct the evaluation.

\subsection{Statistical Analysis}

We used Statistical Packages of Social Sciences (SPSS) software (version 22.0) to analyze the data recorded, while the spondylolisthetic and spino-pelvic parameters were measured through Picture Archiving and Communication System (PACS). The inter-observer agreement of these parameters was expressed by interclass correlation coefficient (ICC).[19] ICC ranged from 0 to 1 , and higher values suggested better agreement. Levels of agreement for ICC were divided into 3 grades, with ICC values 0.00 to 0.40 considered poor agreement, 0.40 to 0.74 fair to good agreement, and 0.75 to 1.00 excellent agreement.[20, 21]

After the normality test, the data that obeys or approximately obeys the normal distribution is represented by mean \pm standard deviation. The differences in imaging parameters and quality of life assessment between the two groups were compared by independent-sample t-test. Meanwhile, $p$ values of $<0.05$ were considered statistically significant for all the above. 


\section{Results}

A total of 108 consecutive cases were involved in the current study, including 26 males and 82 females, with an average age of $61.3 \pm 10.7$ years (range from 48 to 79 years). There were 77 cases of grade I spondylolisthesis, 31 cases belonged to grade II, and 18 cases of kyphotic spondylolisthesis. The age of kyphotic group was significantly higher than that of non-kyphotic group $(67.1 \pm 13.6$ years vs $58.6 \pm 11.9$ years, $t=2.33, p<0.05)$, and the other general data had no statistical significance $(p>0.05) .73$ patients had intractable low back pain, 85 patients had radiating pain in lower extremities, and 80 patients had intermittent claudication. Ultimately, 57 patients underwent surgical operation since they failed to conservative treatment (Table 1).

Table 1

General Information of Patients

\begin{tabular}{|lll|}
\hline No. & Content & Number \\
\hline $\mathbf{1}$ & Sex (Male / Female) & $26 / 82$ \\
\hline $\mathbf{3}$ & Age (Kyphotic / Non-kyphotic) & $61.3 \pm 10.7(67.1 \pm 13.6 / 58.6 \pm 11.9)$ \\
\hline $\mathbf{4}$ & Kyphotic / Non-kyphotic & $18 / 90$ \\
\hline & Meyerding classification & \\
\hline & Grade 1 & 31 \\
\hline $\mathbf{5}$ & Clinical symptoms & \\
\hline & Intractable low back pain & 73 \\
\hline & Radiating pain in lower extremities & 85 \\
\hline & Intermittent claudication & 80 \\
\hline 6 & Surgical / Non-surgical treatment & $57 / 51$ \\
\hline & Kyphotic (Surgical / Non-surgical) & $16 / 2$ \\
\hline & Non-kyphotic (Surgical / Non-surgical) & $41 / 49$ \\
\hline
\end{tabular}

\subsection{Inter-observer Reliability}

The inter-observer agreement of the spondylolisthetic and spino-pelvic parameters between the 2 spine surgeons were entirely excellent, and analysis for each parameter resulted in ICC values as follows: 0.795 , $0.854,0.766,0.821,0.847,0.806,0.879,0.902,0.843,0.818,0.847$ and 0.852 , respectively. The results were considered statistically significant $(p<0.01)$ (Table 2$)$. 
Table 2

Inter-observer Reliability of Each Parameter between Spine Surgeons

\begin{tabular}{|c|c|c|c|c|}
\hline \multirow[t]{2}{*}{ Parameters } & \multicolumn{2}{|c|}{ Spine surgeon } & \multicolumn{2}{|c|}{ Inter-observer reliability } \\
\hline & $A^{*}($ Mean $\pm S D)$ & $B^{*}($ Mean $\pm S D)$ & ICC & $\mathrm{p}$ \\
\hline \multicolumn{5}{|c|}{ Spondylolisthetic } \\
\hline SD (\%) & $19.1 \pm 5.8$ & $20.3 \pm 6.7$ & 0.795 & $0.005^{\dagger}$ \\
\hline $\operatorname{SA}\left({ }^{\circ}\right)$ & $6.5 \pm 4.1$ & $6.2 \pm 2.7$ & 0.854 & $<0.001^{\dagger}$ \\
\hline $\mathrm{ADH}(\mathrm{mm})$ & $9.9 \pm 5.3$ & $9.2 \pm 6.0$ & 0.766 & $0.008^{\dagger}$ \\
\hline PDH (mm) & $7.6 \pm 4.5$ & $8.0 \pm 5.9$ & 0.821 & $0.002^{\dagger}$ \\
\hline $\operatorname{SAM}\left({ }^{\circ}\right)$ & $3.1 \pm 1.6$ & $2.9 \pm 1.1$ & 0.847 & $<0.001^{\dagger}$ \\
\hline SDM (\%) & $6.9 \pm 3.2$ & $6.4 \pm 2.8$ & 0.806 & $0.003^{\dagger}$ \\
\hline \multicolumn{5}{|l|}{ Spino-pelvic } \\
\hline LL $\left(^{\circ}\right)$ & $47.5 \pm 16.1$ & $46.3 \pm 18.6$ & 0.879 & $<0.001^{\dagger}$ \\
\hline $\operatorname{SS}\left({ }^{\circ}\right)$ & $38.4 \pm 17.1$ & $37.4 \pm 19.8$ & 0.902 & $<0.001^{\dagger}$ \\
\hline $\mathrm{PT}\left({ }^{\circ}\right)$ & $16.1 \pm 9.2$ & $16.5 \pm 8.5$ & 0.843 & $<0.001^{\dagger}$ \\
\hline $\mathrm{PI}\left({ }^{\circ}\right)$ & $54.5 \pm 12.4$ & $52.9 \pm 13.6$ & 0.818 & $0.002^{\dagger}$ \\
\hline $\mathrm{TK}\left({ }^{\circ}\right)$ & $24.7 \pm 10.3$ & $23.8 \pm 11.0$ & 0.847 & $<0.001^{\dagger}$ \\
\hline SVA (mm) & $25.1 \pm 12.3$ & $24.3 \pm 13.5$ & 0.852 & $<0.001^{\dagger}$ \\
\hline
\end{tabular}

\subsection{Spondylolisthetic And Spino-pelvic Parameters}

The spondylolisthetic parameters of the two groups were measured as shown in Table 3. Compared with non-kyphotic group, kyphotic group had larger SA, SD, SDM and SAM. The ADH in kyphotic group was significantly lower than that in non-kyphotic group, while the PDH was significantly higher in non-kyphotic group. All the above differences had statistical significance $(p<0.05)$.

\section{Table 3.}

\section{Pairwise Differences of Spondylolisthetic Parameters between the Two Groups}




\begin{tabular}{lcccc}
\hline गondylolisthetic Parameter & $\begin{array}{c}\text { Kyphotic group } \\
(\text { Mean } \pm \text { SD) }\end{array}$ & $\begin{array}{c}\text { Non-kyphotic group } \\
\text { (Mean } \pm \text { SD) }\end{array}$ & $\begin{array}{c}\text { Pairwise difference } \\
\text { (Mean } \pm \text { SD) }\end{array}$ & $p$ value \\
\hline iD $(\%)$ & $23.1 \pm 7.2$ & $18.5 \pm 5.9$ & $3.6 \pm 4.2$ & $0.032^{*}$ \\
iA $\left({ }^{\circ}\right)$ & $-3.7 \pm 2.8$ & $7.9 \pm 4.5$ & $-11.6 \pm 3.8$ & $<0.001^{*}$ \\
iDH $(\mathrm{mm})$ & $6.8 \pm 4.3$ & $10.1 \pm 6.2$ & $-3.3 \pm 2.7$ & $0.014^{*}$ \\
'DH $(\mathrm{mm})$ & $8.5 \pm 5.7$ & $7.2 \pm 5.0$ & $1.3 \pm 2.1$ & $0.027^{*}$ \\
iAM $\left({ }^{\circ}\right)$ & $4.5 \pm 2.1$ & $2.3 \pm 1.7$ & $2.2 \pm 1.0$ & $<0.001^{*}$ \\
iDM $(\%)$ & $11.2 \pm 4.4$ & $4.7 \pm 3.6$ & $6.5 \pm 4.7$ & $<0.001^{*}$ \\
\hline
\end{tabular}

*The difference of parameter between kyphotic and non-kyphotic group was significant.

The measurements of spino-pelvic parameters were shown in Table 4. The PT and SVA were significantly higher in kyphotic group than in non-kyphotic group, while the SS and LL were significantly lower than those in non-kyphotic group. These results were also considered statistically significant $(p<0.05)$. There was no significant difference in PI and TK between the two groups $(p>0.05)$.

Table 4.

Pairwise Differences of Spino-pelvic Parameters between the Two Groups

\begin{tabular}{lcccc}
\hline jino-pelvic Parameter & $\begin{array}{c}\text { Kyphotic group } \\
(\text { Mean } \pm \text { SD) }\end{array}$ & $\begin{array}{c}\text { Non-kyphotic group } \\
(\text { Mean } \pm \text { SD) }\end{array}$ & $\begin{array}{c}\text { Pairwise difference } \\
(\text { Mean } \pm \text { SD) }\end{array}$ & $p$ value \\
\hline $\mathrm{L}\left({ }^{\circ}\right)$ & $42.1 \pm 11.3$ & $51.7 \pm 14.6$ & $-9.6 \pm 5.3$ & $<0.001^{*}$ \\
is $\left(^{\circ}\right)$ & $35.4 \pm 12.6$ & $40.3 \pm 10.1$ & $-4.9 \pm 4.2$ & $0.013^{*}$ \\
'T $\left(^{\circ}\right)$ & $20.7 \pm 9.5$ & $16.4 \pm 7.6$ & $4.3 \pm 2.9$ & $0.008^{*}$ \\
'I $\left({ }^{\circ}\right)$ & $58.3 \pm 15.4$ & $56.5 \pm 13.2$ & $1.8 \pm 4.7$ & 0.217 \\
'K $\left(^{\circ}\right)$ & $23.8 \pm 10.2$ & $25.9 \pm 8.7$ & $-2.1 \pm 3.2$ & 0.154 \\
iVA $(\mathrm{mm})$ & $36.2 \pm 14.3$ & $17.4 \pm 17.8$ & $18.8 \pm 10.1$ & $<0.001^{*}$ \\
\hline
\end{tabular}

*The difference of parameter between kyphotic and non-kyphotic group was significant.

\subsection{Quality Of Life}

The quality of life assessment results were shown in Table 5. The ODI score, VAS score of low back pain and VAS score of lower extremity pain in kyphotic group were significantly higher than those in nonkyphotic group $(p<0.05)$. 
Table 5

Comparison of Life Quality of Patient between the Two Groups

\begin{tabular}{|lllll|}
\hline Scale & \multicolumn{1}{l}{ Kyphotic group } & Non-kyphotic group & Pairwise difference & \multirow{2}{*}{$\boldsymbol{p \text { value }}$} \\
\cline { 2 - 4 } & (Mean \pm SD) & (Mean \pm SD) & (Mean \pm SD) & \\
\hline ODI score & $50.4 \pm 9.6$ & $43.9 \pm 12.1$ & $6.5 \pm 7.2$ & $0.004 *$ \\
\hline VAS back pain score & $7.1 \pm 2.3$ & $5.5 \pm 1.8$ & $1.6 \pm 0.6$ & $<0.001 *$ \\
\hline VAS leg pain score & $6.8 \pm 2.5$ & $6.0 \pm 2.2$ & $0.8 \pm 0.5$ & $0.023^{*}$ \\
\hline *The difference of parameter between kyphotic and non-kyphotic group was significant. & \\
\hline
\end{tabular}

\section{Discussion}

Our research found that $16.7 \%$ of patients with L4/5 single segment DLS had kyphosis, which was similar to the previous study.[22] The typical imaging manifestation of kyphotic spondylolisthesis is the collapse of disc in the anterior intervertebral space of the spondylolisthetic segment, which may be related to the insufficient support of anterior column caused by the vacuum of disc and the instability of vertebral body led by severe intervertebral disc degeneration (IDD). [8, 23] Some scholars believe that in the early stage of lumbar IDD, the stress concentrates in the anterior intervertebral space and the speed of IDD at the stress-concentrated area increases significantly, which leads to the anterior height collapse and the loss of lordosis of the intervertebral space.[24] Our study found that the PI of patients with DLS was significantly higher than that of normal people,[25] which was consistent with previous research conclusions.[5, 26] Higher PI results in greater stress in the lumbosacral area,[27] which will lead to a significant increase of pressure in $L 4 / 5$ intervertebral space and facet joint. Degeneration acceleration, asymmetry and more prone to sagittal orientation of facet joints will increase the instability.[28, 29] Moreover, IDD and reduction of tensile strain capacity of nucleus pulposus and annulus fibrosus will lead to the intervertebral slippage.[30, 31] The change of LL plays an important role in the pathogenesis of DLS. Chen et al.[4] have indicated that the loss of $A D H$ is an independent risk factor for the occurrence and progression of spondylolisthesis. IDD and spondylolisthesis continue to progress with age.[1] Our study found that the age of the DLS patients with kyphosis was significantly higher than that of nonkyphotic group, and the degree of IDD and anterior intervertebral space collapse were more severe, which led to the slightly higher SD of patients in kyphotic group, and that might be an important factor of kyphotic spondylolisthesis.

According to our results, PT and SVA were larger in patients with kyphosis, while LL and SS were significantly lower than those in non-kyphotic group. Previous studies mostly focused on the morphological characteristics of the spine-pelvis sagittal alignment in patients with DLS. The correlation between the abnormal anatomical sequence of spondylolisthetic segment and the spino-pelvic sagittal morphological changes was often paid less attention.[32, 33] Ferrero et al.[5] analyzed the spinal sagittal 
morphology of 654 patients with DLS in L4/5 segment, and suggested that the loss of lordosis angle in spondylolisthetic segment was closely related to the morphological changes of spino-pelvic sagittal alignment. Kong et al.[15] conducted a retrospective study on 53 patients with L4/5 DLS who underwent interbody fusion, and analyzed the influence of SA, SD and intervertebral space height on postoperative spino-pelvic sagittal parameters. Their results showed that the improvement of SA was the most critical to correct the deformity of spondylolisthetic segment and restore the normal spinal sagittal alignment. However, our results indicated that the soft tissue around the spondylolisthetic vertebrae should be fully released to improve the reduction of spondylolisthesis, and large fusion cage should be implanted to restore intervertebral space height and normal Cobb angle, thus to reconstruct the normal sagittal alignment and achieve better clinical effect. The normal L4/5 intervertebral space is higher in anterior part to maintain LL, however, in the process of IDD, the decrease of ADH will lead to gradual reduction of LL.[24] In addition, the loss of LL aggravates with age, especially after 50.[34] In our study, patients in kyphotic group had significantly older age, more severe loss of ADH and greater reduction of LL, which were bound to cause SVA to move forward. Meanwhile, in order to maintain the sagittal balance, the body needed to rely on pelvic retroversion to compensate, that was, the increase of PT and the decrease of SS. When the LL further decreased and exceeded the compensatory capacity of pelvic retroversion, there might be a tendency of spinal sagittal decompensation, and at that moment, the body might flex the hip and knee to keep upright.[13] This may explain our result that patients with kyphotic DLS in L4/5 segment have more obvious loss of $L L$, pelvic retroversion and trunk anteversion than patients in nonkyphotic group.

In addition, the VAS and ODI scores showed that the life quality of patients with kyphosis at the time of consultation was significantly lower than that of patients in non-kyphotic group. Fritz et al.[35] considered that lumbar instability and neural compression were the primary factors leading to low back pain and dysfunction in patients with DLS. Chen et al.[7] measured the sagittal displacement and rotation of the spondylolisthetic segment through flexion and extension lateral radiographs, and found that patients with kyphotic spondylolisthesis had obvious lumbar instability. Some scholars indicated that local instability caused by the loss of LL was the primary reason leading to clinical symptoms of DLS patients in L4/5 segment. Our study found that the range of motion (ROM) of the spondylolisthetic segment (including SAM, SDM) in patients with kyphosis was significantly higher than that in the non-kyphotic group, which also confirmed the above-mentioned studies, that was, the loss of disc height and the relaxation of peripheral ligaments caused the increase of ROM, thus gradually destabilizing the spine and resulting in more severe back pain and dysfunction in DLS patients with kyphosis.[30, 31] As mentioned previously, pelvic retroversion, trunk anteversion, and hip and knee flexion are compensatory methods for kyphotic DLS patients to maintain spinal sagittal balance. These postures are bound to affect the spino-pelvic biomechanical distribution of patients, resulting in excessive muscle energy consumption, muscle fatigue, and other more severe clinical symptoms.[24, 36]

The current study has several limitations. Firstly, is the relatively small sample size of kyphotic DLS. Further expanding our sample population will eliminate coincidence as much as possible, reduce measuring deviation and allow for more meaningful statistical testing. Secondly, the degree of IDD and 
the facet joint orientation were not compared between the two groups, so the mechanism of kyphotic DLS could not be determined. Finally, only L4/5 single segment, low-grade DLS patients were included in this study, which might make the results relatively one-sided and limited. Therefore, in future clinical work, high-quality, multicenter, large sample and wide case scope studies should be conducted to provide spine surgeons with the best evidence-based information.

\section{Conclusion}

The incidence of kyphotic spondylolisthesis in patients with L4/5 low-grade DLS is $16.7 \%$. Compared with those in non-kyphotic group, patients with kyphosis show inferior results of spondylolisthetic parameters, more severe loss of LL, more obvious pelvic retroversion and trunk anteversion, which indicates worse spino-pelvic sagittal balance. In addition, their quality of life was significantly lower than that of patients in non-kyphotic group. However, larger sample and multi-center studies should be performed to improve the accuracy and reliability of the finding, and further clinical comparison should be conducted in future works.

\section{Abbreviations}

DLS (degenerative lumbar spondylolisthesis)

MR (magnetic resonance)

VAS (visual analogue scale)

ODI (Oswestry disability index)

SD (slip degree)

$\mathrm{ADH}$ (anterior disc height)

PDH (posterior disc height)

SA (slip angle)

SAM (slip angle motion)

SDM (slip degree motion)

LL (lumbar lordosis)

SS (sacral slope)

PT (pelvic tilt)

$\mathrm{PI}$ (pelvic incidence) 
TK (thoracic kyphosis)

SVA (sagittal vertical axis)

ICC (interclass correlation coefficient)

IDD (intervertebral disc degeneration)

ROM (range of motion)

\section{Declarations}

\section{Funding}

No funding was obtained for this study.

\section{Conflict of interest statement}

All authors read and approved the final manuscript and declare that they have no competing interests.

\section{Acknowledgements}

I want to thank my love, Du Ying, no matter how difficult it is, she never gives up, always cares for me, silently supports me, and gives me courage when I lose confidence. Without her help, understanding, tolerance and support, I believe that the life of PhD in these three years will be very different.

\section{Ethics approval and consent to participate}

The case was reviewed by the Longhua Hospital Ethics Committee and ethical approval was waived as written consent was obtained from the patient.

\section{Consent for publication}

Written patient consent was obtained for publication of all aspects of the case including personal and clinical details and images, which may compromise anonymity.

\section{Availability of data and material}

All supporting data can be provided upon request to the authors.

\section{Competing interests}

All authors read and approved the final manuscript and declare that they have no competing interests.

\section{Funding}

No funding was obtained for this study. 


\section{Authors' contributions}

XCQ and YMC are co-first authors of this manuscript. XCQ designed the study and collected the data. YMC did the data analysis. XCQ wrote the manuscript. MW revised the manuscript and decided to submit the manuscript for publication. All authors read and approved the final manuscript.

\section{Acknowledgements}

I want to thank my love, Du Ying, no matter how difficult it is, she never gives up, always cares for me, silently supports me, and gives me courage when I lose confidence. Without her help, understanding, tolerance and support, I believe that the life of a PhD in these three years will be very different.

\section{References}

1. Herman MJ, Pizzutillo PD, Cavalier R. Spondylolysis and spondylolisthesis in the child and adolescent athlete. Orthop Clin N Am. 2003;34(3):461-7.

2. Jacobsen S, Sonne-Holm S, Rovsing H, et al. Degenerative lumbar spondylolisthesis: an epidemiological perspective: the Copenhagen osteoarthritis study. Spine (Phila Pa 1976). 2007;32(1):120-5.

3. Bernhardt M, Bridwell K. Segmental analysis of the sagittal plane alignment of the normal thoracic and lumbar spines and thoracolumbar junction. Spine. 1989;14(7):717-21.

4. Chen IR, Wei TS. Disc height and lumbar index as independent predictors of degenerative spondylolisthesis in middle-aged women with low back pain. Spine. 2009;34(13):1402-9.

5. Ferrero E, Ould-Slimane M, Gille O, et al. Sagittal spinopelvic alignment in 654 degenerative spondylolisthesis. Eur Spine J. 2015;24(6):1219-27.

6. Suda K, Ito M, Abumi K, et al. Radiological risk factors of pseudoarthrosis and/or instrument breakage after PLF with the pedicle screw system in isthmic spondylolisthesis. J Spinal Disord Tech. 2006;19(8):541-6.

7. Chen SY, Lu ML, Niu CC, et al. Results of instrumented posterolateral fusion in treatment of lumbar spondylolisthesis with and without segmental kyphosis: a retrospective investigation. Biomed J. 2015;38(3):262-8.

8. Kepler CK, Hilibrand AS, Sayadipour A, et al. Clinical and radiographic degenerative spondylolisthesis (CARDS) classification. Spine J. 2015;15(8):1804-11.

9. Huang $\mathrm{K}$, Lin R, Lee $\mathrm{Y}$, et al. Factors affecting disability and physical function in degenerative lumbar spondylolisthesis of L4-5: evaluation with axially loaded MRI. Eur Spine J. 2009;18(12):1851-7.

10. Liao J, Lu M, Niu C, et al. Surgical outcomes of degenerative lumbar spondylolisthesis with anterior vacuum disc: Can the intervertebral cage overcome intradiscal vacuum phenomenon and enhance posterolateral fusion? J Orthop Sci. 2014;19(6):851-9. 
11. Matsunaga S, Sakou T, Morizono Y, et al. Natural history of degenerative spondylolisthesis. Pathogenesis and natural course of the slippage. Spine. 1990;15(11):1204-10.

12. Labelle H, Roussouly $P$, Berthonnaud E, et al. Spondylolisthesis, pelvic incidence, and spinopelvic balance: a correlation study. Spine (Phila Pa 1976). 2004;29(18):2049-54.

13. Labelle H, Mac-Thiong JM, Roussouly P. Spino-pelvic sagittal balance of spondylolisthesis: a review and classification. Eur Spine J. 2011;20(suppl 5):641-6.

14. Meyerding HW. Spondylolisthesis. Surg Gynecol Obstet. 1932;54:371-7.

15. Kong $L$, Zhang $Y$, Wang F, et al. Radiographic restoration of sagittal spinopelvic alignment after posterior lumbar interbody fusion in degenerative spondylolisthesis. Clin Spine Surg. 2016;29(2):8792.

16. Tarpada SP, Cho W, Chen F, et al. Utility of Supine Lateral Radiographs for Assessment of Lumbar Segmental Instability in Degenerative Lumbar Spondylolisthesis. Spine. 2018;43(18):1275-80.

17. Duval-Beaupère $G$, Schmidt $C$, Cosson $P$. A barycentremetric study of the sagittal shape of spine and pelvis: the conditions required for an economic standing position. Ann Biomed Eng. 1992;20(4):45162.

18. Mac-Thiong JM, Berthonnaud E, DimarJN, et al. Sagittal alignment of the spine and pelvis during growth. Spine (PhilaPa1976). 2004;29(15):1642-1647.

19. Fleiss J. Measuring nominal scale agreement among many raters. Psycho Bull. 1971;76:378-81.

20. Cohen J. A coefficient of agreement for nominal scales. Educ Psychol Meas. 1960;20:37-46.

21. Landis JR, Koch GG. The measurement of observer agreement for categorical data. Biometrics. 1977;33(1):159-74.

22. Sun $X$, Chen X, Li S, et al. Transforaminal lumbar interbody fusion in treating degenerative spondylolisthesis at L4 with kyphotic versus non-kyphotic angulation. Chin J Orthop. 2017;37(20):1249-55.

23. Lin T, Liao J, Tsai T, et al. The effects of anterior vacuum disc on surgical outcomes of degenerative versus spondylolytic spondylolisthesis: at a minimum two-year follow-up. BMC Musculoskelet Disord. 2014;15(1):329-37.

24. Xu Y, Guo Z, Yun C, et al. Morphology and significance of lumbar disc in lumbar degenerative spondylolisthesis plus lumber disc herniation. Chinese Journal of Spine Spinal Cord. 2012;22(5):398-400.

25. Zhu Z, Xu L, Zhu F, et al. Sagittal alignment of spine and pelvis in asymptomatic adults. Spine. 2014;39(1):E1-6.

26. Funao $\mathrm{H}$, Tsuji T, Hosogane $\mathrm{N}$, et al. Comparative study of spinopelvic sagittal alignment between patients with and without degenerative spondylolisthesis. Eur Spine J. 2012;21(11):2181-7.

27. Barrey C, Jund J, Perrin G, et al. Spinopelvic alignment of pelvic with degenerative spondylolisthesis. Neurosurgery. 2007;61(5):981-6. 
28. Berlemann U, Jeszenszky DJ, Buhler DW, et al. The role of lumbar lordosis, vertebral end-plate inclination, disc height, and facet orientation in degenerative spondylolisthesis. J Spinal Disord. 1999;12(1):68-73.

29. Xu C, Yin M, Mo W. Correlation and differences in lumbopelvic sagittal alignment parameters between lumbar radiographs and magnetic resonance images. Global Spine J. 2020;2192568220947049.

30. Chen X, Zhou Q, Xu L, et al. Does kyphotic configuration on upright lateral radiograph correlate with instability in patients with degenerative lumbar spondylolisthesis? Clin Neurol Neurosurg. 2018;173:96-100.

31. Abu-Leil S, Floman Y, Bronstein Y, et al. A morphometric analysis of all lumbar intervertebral discs and vertebral bodies in degenerative spondylolisthesis. Eur Spine J. 2016;25(8):2535-45.

32. Zhou Z, Hou C, Li D, et al. The pelvic radius technique in the assessment of spinopelvic sagittal alignment of degenerative spondylolisthesis and lumbar spinal stenosis. J Orthop Sci. 2018;23(6):902-7.

33. Bae JS, Jang JS, Lee $\mathrm{SH}$, et al. Radiological analysis of lumbar degenerative kyphosis in relation to pelvic incidence. Spine J. 2012;12(11):1045-51.

34. Uehara M, Takahashi J, Ikegami S, et al. Sagittal spinal alignment deviation in the general elderly population: A Japanese cohort survey randomly sampled from a basic resident registry. Spine J. 2019;19(2):349-56.

35. Fritz JM, Delitto A, Welch WC, et al. Lumbar spinal stenosis: a review of current concepts in evaluation, management, and outcome measurements. Arch Phys Med Rehabil. 1998;79(6):700-8.

36. Liu F, Wang W, Weng W, et al. A study on the sagittal alignment of the spinal-pelvic-lower leg and its clinical relevance in patients with knee osteoarthritis. Orthopedic Journal of China. 2015;23(9):7849.

\section{Figures}



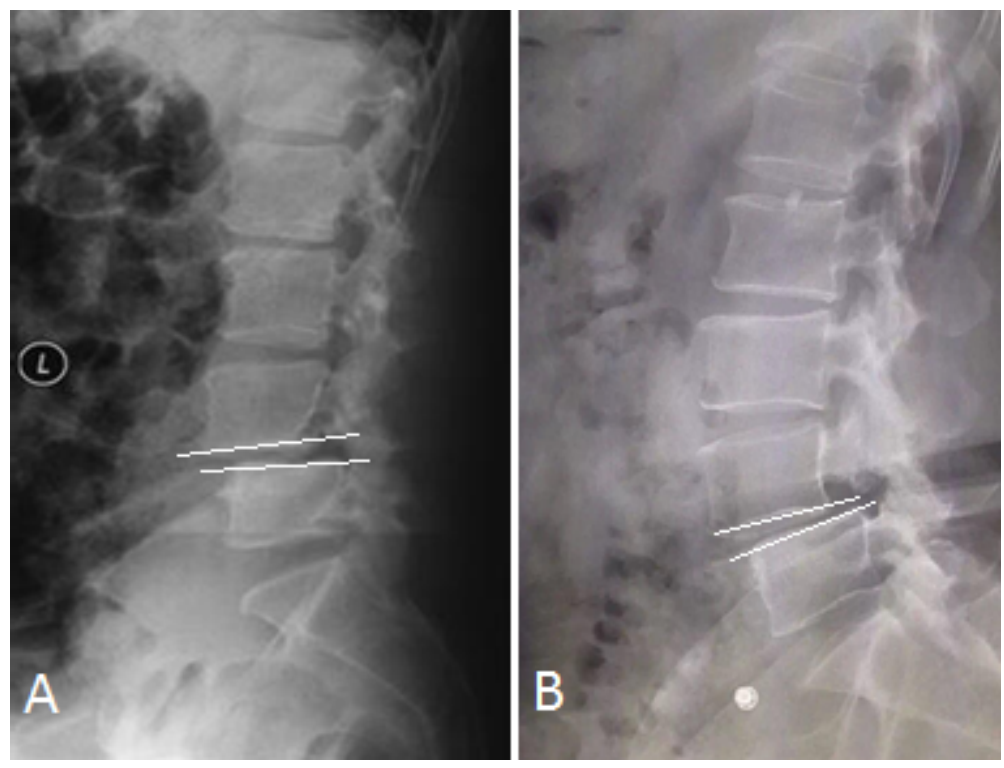

\section{Figure 1}

Illustrative radiographs of kyphotic (A) and non-kyphotic (B) DLS.

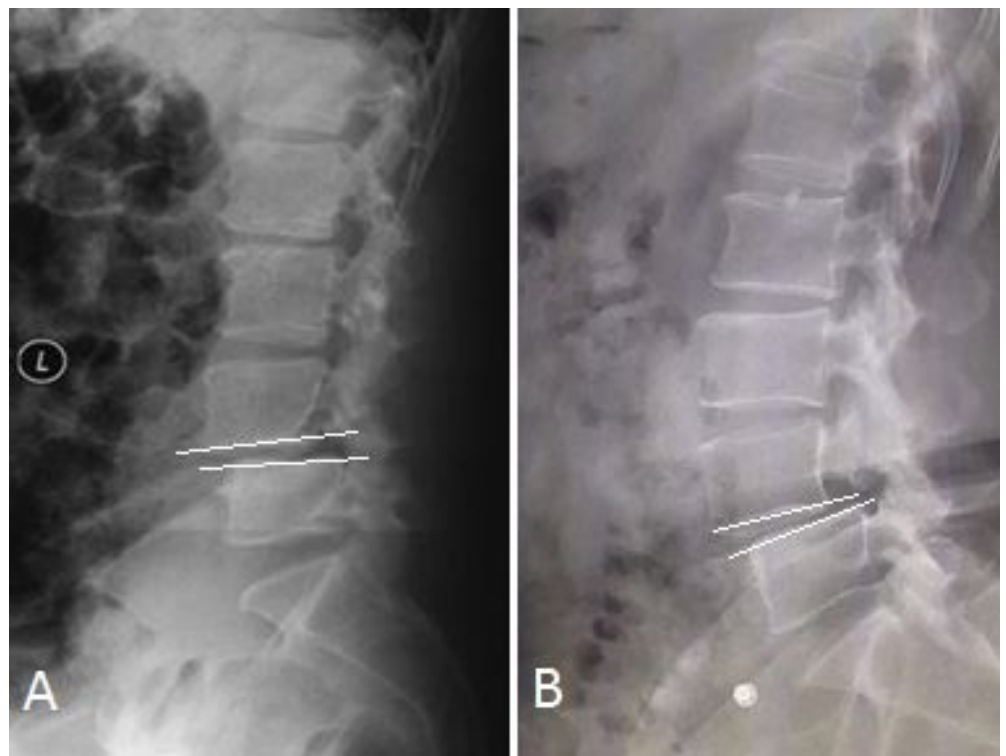

\section{Figure 1}

Illustrative radiographs of kyphotic (A) and non-kyphotic (B) DLS. 


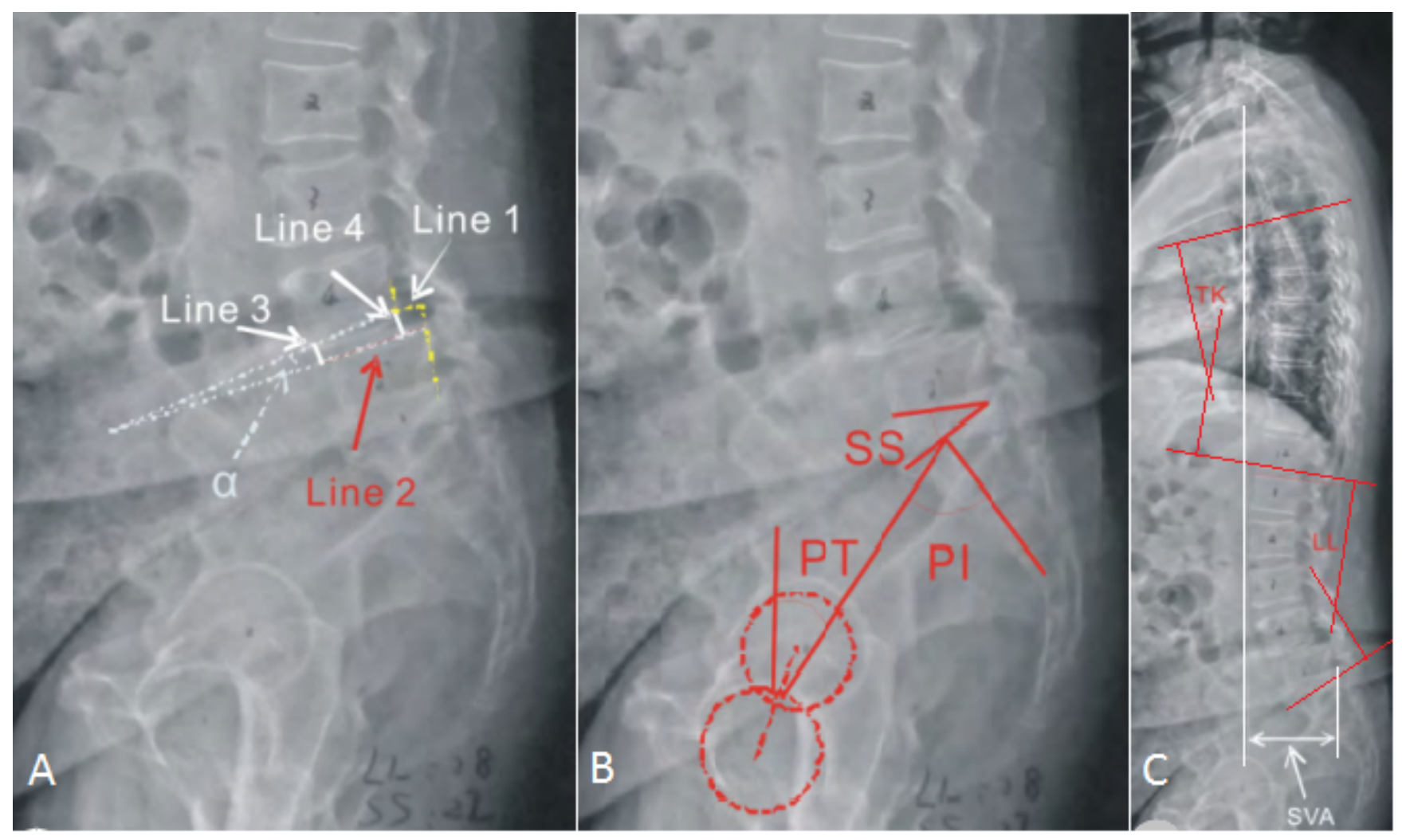

\section{Figure 2}

Radiological parameters. (A) Spondylolisthetic parameters: SD [(line 1)/(line 2)×100\%], SA (a), ADH (line 3/line 2), PDH (line 4/line 2); (B) and (C) Spino-pelvic sagittal parameters: PI, PT, SS, LL, TK, and SVA.

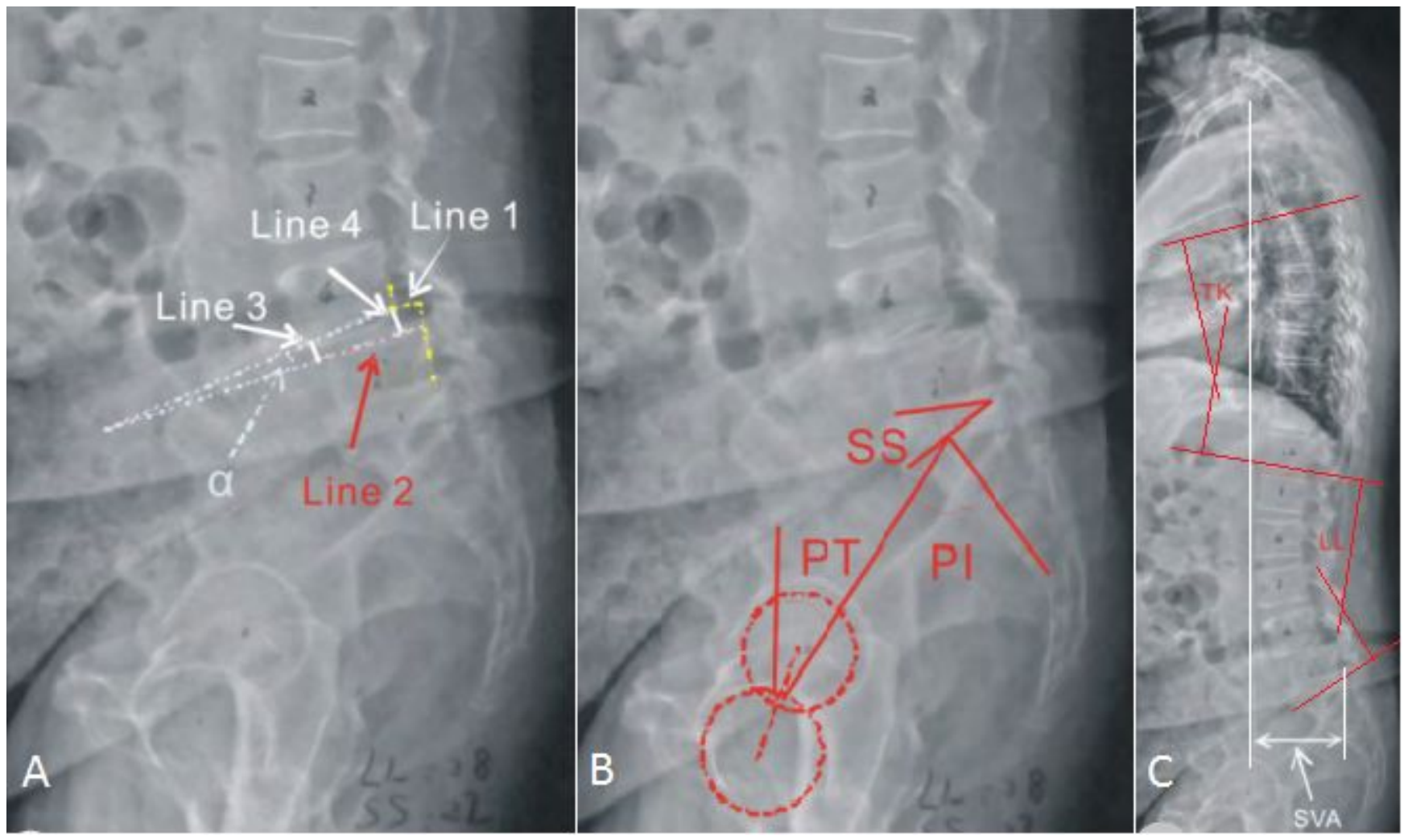

Figure 2 
Radiological parameters. (A) Spondylolisthetic parameters: SD [(line 1)/(line 2)×100\%], SA (a), ADH (line 3/line 2), PDH (line 4/line 2); (B) and (C) Spino-pelvic sagittal parameters: PI, PT, SS, LL, TK, and SVA. 